\title{
Electroconvulsive therapy and later stroke in patients with affective disorders
}

Maarten Pieter Rozing, Martin Balslev Jørgensen and Merete Osler

\section{Summary}

The long-term effects of electroconvulsive therapy (ECT) on the risk of stroke are unknown. We examined the association between ECT and risk of incident or recurrent stroke. A cohort of 174534 patients diagnosed with affective disorder between 2005 and 2016 in the Danish National Patient Registry were followed for stroke until November 2016. The association between ECT and stroke was analysed using cox regression with multiple adjustment and propensity-score matching on sociodemographic and clinical variables. In 162595 patients without previous stroke, 5781 (3.6\%) were treated with ECT. The total number of patients developing stroke during follow-up was 3665, of whom 165 had been treated with ECT. In patients $<50$ years, ECT was not associated with stroke (adjusted hazard ratio $(H R)=1.29$, $95 \% \mathrm{Cl}$ 0.87-1.93). In patients $\geq 50$, ECT was associated with a lower risk of stroke (adjusted $\mathrm{HR}=0.69,95 \% \mathrm{Cl} 0.57-0.89$ ), but this estimate was likely influenced by competing mortality risk. Of 11939 patients with a history of stroke, $228(1.9 \%)$ were treated with ECT. During follow-up, 2330 (19.5\%) patients had a recurrence, of which 26 were patients treated with ECT. ECT was not associated with risk of a new event $(\mathrm{HR}=0.69,95 \% \mathrm{Cl} 0.46-$ $1.00 ; P=0.05)$. ECT is not associated with an elevated risk of incident or recurrent stroke.

\section{Declaration of interest}

None.

\section{Keywords}

Electroconvulsive therapy; clinical neurology; epidemiology.

\section{Copyright and usage}

(c) The Royal College of Psychiatrists 2018.
Electroconvulsive therapy (ECT) is a generally well-tolerated and effective treatment for mood disorders. ${ }^{1}$ Most neurological adverse effects of ECT concern amnesia, headache, disorientation and transient post-ictal neurological deficits. The substantial fluctuations in cardiovascular parameters and alteration in brain perfusion during ECT administration could theoretically elicit a stroke. ${ }^{2}$ However, true cerebrovascular complications directly following ECT are an extremely rare complication according to series of case reports. ${ }^{3,4}$ However, long-term effects of repeated administration of ECT on stroke risks have not been investigated. We examined the association between ECT and long-term risk of incident stroke (including both cerebrovascular accidents and transient ischaemic attacks (TIA)) in patients with a first-time hospital diagnosis of affective disorder. Since ECT may be used in patients with antidepressant resistance with post-stroke depression, ${ }^{5}$ we also explored the frequency of ECT and associated risk of recurrence in patients with a history of stroke.

\section{Method}

\section{Study population}

All citizens in Denmark with a first-time hospital contact for an affective disorder from 1 January 2005 to 31 December 2015 were included in this cohort study. In total, 174534 in- or out-patients above 10 years were identified by record linkage with the Danish National Patient Registry (DNPR) ${ }^{6}$ using ICD-8 or ICD-10 (432434,436 and F30.0-F39.9). ${ }^{7,8}$ Of them 11939 had been diagnosed with stroke before or at study entry according to the DNPR, which was screened for all admissions because of intracerebral haemorrhagic stroke (431; I61), ischaemic stroke $(432 ; 433 ; 434$; I63), unspecified stroke (I64) and TIA (436; G45) occurring between January 1979 and December 2015. In the present study, ischaemic strokes and unspecified strokes were analysed together because Krarup et al ${ }^{9}$ have shown that most unspecified stroke diagnosis in the DNPR actually are ischaemic strokes. The study was approved by the Danish Data Protection Agency.

\section{Electroconvulsive therapy (exposure)}

From the DNPR we retrieved information on all ECTs registered for patients and treatment was defined at the time of first ECT registration. The relevant codes and classifications have been described previously. ${ }^{6,10}$ The number of ECT sessions was counted from study entry until censoring, and dichotomised ( $\leq 10$ or $>10$ sessions) based on the median number of ECT sessions necessary for remission. ${ }^{10}$

\section{Subsequent stroke (outcomes)}

All patients were followed for admissions with stroke as main diagnosis (ICD-10: I61; I63; I64 and G45) from date of inclusion until end of October 2016. For patients with a previous stroke a reevent was defined as a new admission within at least 28 days after the last event as recommended in a previous Danish study. ${ }^{11}$ However, since many re-events occur during the first month, ${ }^{12}$ we repeated the analyses using a less conservative definition by counting all readmissions as a result of stroke occurring after 7 days.

\section{Covariates}

The following covariates were included based on a literature review on factors associated with selection of patients for ECT and risk of stroke: gender, age, level of education, comorbid alcohol misuse, hypertension, coronary heart disease, obesity and diabetes. (see supplementary material for details; available at https://doi.org/10.1192/ bjp.2018.150)

\section{Statistical analysis}

Differences in the distribution of ECT and cerebrovascular events in relation to covariates were examined using the simple chi-square test. The associations of first ECT with recurrent or incident strokes were analysed using Cox proportional hazard regression (hazard ratio (HR) and 95\% CIs) with age as underlying timescale. The analyses of recurrence were based on the 11939 patients with at least one previous stroke before inclusion as a result of an affective disorder diagnosis, whereas the analyses of incidence were based on 
Hazard ratio $(95 \% \mathrm{cl})$

All

\section{Crude}

Adjusteda

Propensity-score matched

$<$ Age 50

Crude

Adjusteda

Propensity-score matched

$\geq$ Age 50

Crude

Adjusteda

Propensity-score matched
$0.70(0.60-0.84)$

$0.79(0.66-0.94)$

$0.81(0.64-1.03)$

$1.20(0.81-1.77)$

$1.29(0.87-1.93)$

$1.43(0.78-2.60)$

$0.66(0.50-0.86)$

$0.69(0.57-0.89)$

$0.73(0.57-0.95)$

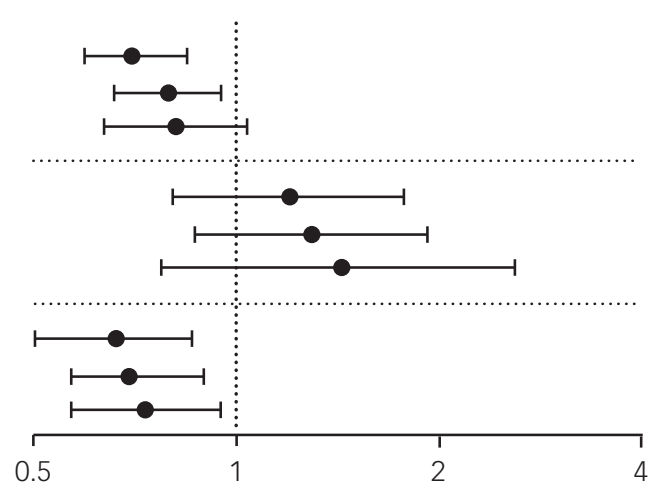

Fig. 1 Associations (hazard ratios and $95 \% \mathrm{Cl}$ ) between electroconvulsive therapy and stroke in relation to age at study entry in a cohort of 162595 patients diagnosed with affective disorders from 2005 to 2015 in Denmark.

a. Adjusted for gender, educational level, subdiagnosis, comorbid hypertension, coronary heart disease, diabetes, obesity and alcohol misuse.

the remaining 162595 patients. To preclude potential immortaltime bias, ECT was entered as a time-dependent variable by splitting the data-set according to time of first ECT. Patients were followed from age at study entry (date of first registered affective disorder) until the age of first registration of stroke, emigration, death or end of follow-up (November 2016), whichever came first.

We also performed a propensity-score calibration matching with a one-to-one match on the nearest neighbour. The propensity score was estimated as the relative hazard of the exposure (ECT) conditional on the baseline covariates in a Cox regression. Competing mortality risks were accounted for by using the FineGray competing risks regression by means of the stcrreg command in Stata, which specifies a model for the cumulative incidence and generates subdistribution hazard ratios (SHRs). Since, this approach is not appropriate when the exposure is time varying, it was only used in the propensity-score matched sample, where patients were entered at time of ECT. To account for acute mortality we also repeated the analyses with a lag period of 1 and 2 years after study entry. The proportional hazard assumption was examined graphically and showed no signs of violation. We performed various additional analyses. First, we excluded TIA diagnoses and restricted the analyses to in-patient diagnoses. Second, we restricted the analyses to 30 and 180 days post-ECT to assess the short-term risk of stroke after ECT (For results see supplementary material). The analyses were performed in Stata_14.

\section{Results}

\section{ECT and incident stroke}

In the 162595 patients with no history of previous stroke, 5781 (3.6\%) were treated with ECT (supplementary Table 1). The mean follow-up was 5.1 years (range 0.1-12.0) during which 3665 patients had a first-time stroke diagnosis registered in the DNPR (incidence rate (IR) 42.8/10 000 person-years, 95\% CI 41.5-44.3). In all patients the age-standardised incidence of stroke was slightly lower among patients treated with ECT $(n=165, \mathrm{IR}=38.8 / 10000$ person-years) than those not treated with ECT $(n=3500,42.7 /$ 10000 person-years). This difference varied in relation to age. Figure 1 gives the crude and adjusted hazard ratios (HR) for patients $\leq 50$ or $\geq 50$. In the younger patients, ECT was not associated with stroke ( $\mathrm{HR}=1.20,95 \% \mathrm{CI} 0.81-1.77)$, whereas in the older patients ECT was associated with a lower risk of stroke before and after adjustments ( $\mathrm{HR}=0.66,95 \% \mathrm{CI} 0.50-0.86)$. However, the SHR suggested that the estimates for elderly people might be influenced by the presence of competing mortality risk $(\mathrm{SHR}=0.86,95 \% \mathrm{CI}$ $0.67-1.10)$.

Among the patients treated with ECT, 2755 (47.7\%) had more than ten sessions and this was not associated with risk of incident stroke neither before ( $\mathrm{HR}=0.86,95 \%$ CI $0.61-1.20)$ or after (HR $=0.86,95 \%$ CI $0.61-1.20)$ adjustments. The HRs did not change materially when follow-up was started 1 or 2 years after study entry. Neither did the results change when TIA and out-patients diagnoses were excluded (data not shown).

\section{ECT and recurrence}

Of the patients with a previous stroke, 228 (1.9\%) were treated with ECT (supplementary Table 1). ECT was most common among those with a previous TIA or who had only one previous stroke. During follow-up, 2330 (19.5\%) patients had a recurrent stroke following the definition of 28 days or more from latest event after inclusion, yielding a rate of 62.9 (95\% CI 60.4-65.5) events per 1000 person-years with the highest rate in patients $>70$ years (supplementary Table 1) and among those with $<1$ year since last stroke.

Of the 2330 patients who had a new event, 26 occurred among patients treated with ECT. In patients treated with ECT the risk of recurrence was lower than in non-ECT patients $(\mathrm{HR}=0.61,95 \% \mathrm{CI}$ 0.41-0.91), but the association was attenuated after adjustment for covariates $(\mathrm{HR}=0.69,95 \% \mathrm{CI} 0.46-1.00 ; P=0.05)$. Among those treated with ECT $44.3 \%$ had $>10$ sessions and this was not associated with risk of recurrent stroke. The results were similar when readmissions occurring after 7 days were included.

\section{Discussion}

This register-based cohort study of patients with affective disorders showed that ECT was not associated with stroke incidence or stroke recurrence. A presumed reduced risk of incident stroke in older patients treated with ECT might be explained by competing mortality risk. 
Our findings align with previous studies, predominantly case reports showing that stroke is an exceptionally rare complication of ECT. ${ }^{3,4}$ In the present study we extended these findings by showing that ECT was also not associated with an elevated risk during long-term follow-up. This is remarkable since ECT is often used in elderly people with treatment-refractory depression where vascular pathology is commonly involved in the pathogenesis. ${ }^{13}$ Perhaps the vascular pathology in vascular depression is not in itself a risk factor for stroke.

Arguably, selection bias may have influenced our findings. Plausibly the health status of the patient has played a role in the decision to administer ECT. ${ }^{14}$ However, we aimed to reduce this risk of bias by conducting a propensity-score calibration matching. A second limitation of our study could be the variation in the quality of stroke diagnosis. Although three validation studies have shown high predictive values $(<80 \%)$ for overall and ischaemic stroke in the $\mathrm{DNPR}^{9,15,16}$ stroke as a secondary diagnosis among outpatient clinics seem to have low predictive values. ${ }^{15}$ Consequently, we only used stroke as the main diagnosis as our outcome.

The strengths of this study are the large sample size, use of nationwide population-based registers in a country with free access to healthcare and a nearly complete coverage of hospital contacts. The registration of ECT is mandatory and has high completeness. $^{10}$

In conclusion, our findings suggest that ECT as currently practised does not increase the risk of incident or recurrent stroke. Our study provides evidence to inform patients about the long-term risks of having ECT.

Maarten Pieter Rozing, MD, PhD, Assistant Professor, Section of Epidemiology and The Research Unit for General Practice and Section of General Practice, Department of Public Health, and Center for Healthy Aging, University of Copenhagen, Denmark; Martin Balslev Jørgensen, MD, PhD, Clinical Professor, Psychiatric Centre Copenhagen Department O, Rigshospitalet, Denmark; Merete Osler, MD, PhD, Clinical Professor, Section of Epidemiology, Department of Public Health, University of Copenhagen, Center for Clinical Research and Prevention, Bispebjerg and Frederiksberg Hospitals and Danish Ageing Research Center, Department of Public Health, University of Southern Denmark, Denmark

Correspondence: Maarten Pieter Rozing, Øster Farimagsgade 5, P.O. 2099, 1014, København K, Denmark. Email: mroz@sund.ku.dk

First received 15 Mar 2018, final revision 18 Jun 2018, accepted 24 Jun 2018

\section{Funding}

The work was supported by the Danish Council for Independent Research, grant-ID: DFF-61100195.

\section{Supplementary material}

Supplementary material is available online at http://dx.doi.org/10.1192/bjp.2018.150.

\section{References}

1 Carney S, Cowen P, Geddes J, Goodwin G, Rogers R, Dearness K.. et al. UK ECT Review Group. Efficacy and safety of electroconvulsive therapy in depressive disorders: a systematic review and meta-analysis. Lancet 2003; 361: 799-808.

2 Nagler J. Heart rate changes during electroconvulsive therapy. Ann Gen Psychiatry 2013; 12: 19.

3 Lee K. Acute embolic stroke after electroconvulsive therapy. J ECT 2006; 22 67-9.

4 Bruce BB, Henry ME, Greer DM. Ischemic stroke after electroconvulsive therapy. J ECT 2006; 22: 150-2.

5 Rabheru K. The use of electroconvulsive therapy in special patient populations. Can J Psychiatry 2001; 46: 710-9.

6 Schmidt M, Schmidt SA, Sandegaard JL, Ehrenstein V, Pedersen L, Sorensen HT. The Danish National Patient Registry: a review of content, data quality, and research potential. Clin Epidemiol 2015; 7: 449-90.

7 World Health Organization. International Statistical Classification of Diseases and Related Health Problems (ICD-8). WHO, 1967.

8 World Health Organization. The ICD-10 Classification of Mental and Behavioural Disorders: Clinical Descriptions and Diagnostic Guidelines. WHO, 1992.

9 Krarup LH, Boysen G, Janjua H, Prescott E, Truelsen T. Validity of stroke diagnoses in a National Register of Patients. Neuroepidemiology 2007; 28: 150-4.

10 Hundrup E, Osler $\mathrm{M}$, Jørgensen $\mathrm{MB}$. Time trends and variations in electroconvulsive treatment in Denmark 2008 to 2014: a nationwide register-based study. J ECT 2017; 33: 243-8.

11 Thorvaldsen P, Asplund K, Kuulasmaa K, Rajakangas AM, Schroll M. Stroke incidence, case fatality, and mortality in the WHO MONICA project. World Health Organization Monitoring Trends and Determinants in Cardiovascular Disease. Stroke 1995; 26: 361-7.

12 Arsava EM, Kim GM, Oliveira-Filho J, Gungor L, Noh HJ, Lordelo Mde J, et al. Prediction of early recurrence after acute ischemic stroke. JAMA Neurol 2016; 73: 396-401.

13 Aizenstein HJ, Baskys A, Boldrini M, Butters MA, Diniz BS, Jaiswal MK, et al. Vascular depression consensus report - a critical update. BMC Med 2016; 14: 161.

14 Kerner N, Prudic J. Current electroconvulsive therapy practice and research in the geriatric population. Neuropsychiatry 2014; 4: 33-54.

15 Luhdorf $\mathrm{P}$, Overvad K, Schmidt EB, Johnsen SP, Bach FW. Predictive value of stroke discharge diagnoses in the Danish National Patient Register. Scand J Public Health 2017; 45: 630-6.

16 Johnsen SP, Overvad K, Sorensen HT, Tjonneland A, Husted SE. Predictive value of stroke and transient ischemic attack discharge diagnoses in The Danish National Registry of Patients. J Clin Epidemiol 2002; 55: 602-7. 